\title{
Hydrogen Permeation in FeCrAl Alloys for LWR Cladding Application ${ }^{\star \star \diamond}$
}

\author{
Xunxiang Hu, ${ }^{1,2}$ Kurt A. Terrani, ${ }^{2}$ Brian D. Wirth, ${ }^{1,2}$ and Lance L. Snead ${ }^{2}$
}

1. Department of Nuclear Engineering, University of Tennessee, Knoxville, TN 37996

2. Oak Ridge National Laboratory, Oak Ridge, TN 37831

\begin{abstract}
FeCrAl, an advanced oxidation-resistant iron-based alloy class, is a highly prevalent candidate as an accident-tolerant fuel cladding material. Compared with traditional zirconium alloy fuel cladding, increased tritium permeation through FeCrAl fuel cladding to the primary coolant is expected, raising potential safety concerns. In this study, the hydrogen permeability of several $\mathrm{FeCrAl}$ alloys was obtained using a static permeation test station, which was calibrated and validated using 304 stainless steel. The high hydrogen permeability of $\mathrm{FeCrAl}$ alloys leads to concerns with respect to potentially significant tritium release when used for fuel cladding in LWRs. The total tritium inventory inside the primary coolant of a light water reactor was quantified by applying a 1-dimensional steady state tritium diffusion model to demonstrate the dependence of tritium inventory on fuel cladding type. Furthermore, potential mitigation strategies for tritium release from $\mathrm{FeCrAl}$ fuel cladding were discussed and indicate the potential for application of an alumina layer on the inner clad surface to serve as a tritium barrier.
\end{abstract}

\footnotetext{
${ }^{9}$ The United States Government retains, and by accepting the article for publication, the publisher acknowledges that the United States Government retains, a non-exclusive, paid-up, irrevocable, worldwide license to publish or reproduce the published form of this work, or allow others to do so, for United States Government purposes.
} 
More effort is required to develop a robust, economical mitigation strategy for tritium permeation in reactors using FeCrAl clad fuel assemblies.

\section{Introduction}

Driven by the need to enlarge the safety margins of nuclear reactors in accident scenarios, research and development (R\&D) of accident-tolerant fuel has become a hot topic in the nuclear engineering and materials community. The FeCrAl class of ferritic alloys, which resist steam oxidation at high-temperatures, have been proposed as candidate accidenttolerant fuel cladding materials for light water reactor (LWR) fuel assemblies [1]. FeCrAl alloys consist of nominally $10-15 \mathrm{wt} \%$ chromium and $3-6 \mathrm{wt} \%$ aluminum dissolved in a body-centered-cubic (BCC) iron lattice, which stabilize the BCC structure to the melting point of the material $\left(\sim 1500^{\circ} \mathrm{C}\right)$. The high-temperature strength [1] and excellent oxidation resistance $[2,3]$ of these alloys delay the onset and extent of ballooning and burst; reduce the heat release and hydrogen generation due to oxidation; and enable operators to maintain a coolable, controllable core for a longer period under design-basis and severe accidents, thus enhancing safety margins under these scenarios. The improvements in fuel bundle performance in accidents must accompany simultaneous improvement (or at least comparable performance) compared with zirconium ( $\mathrm{Zr}$ ) -based alloys under normal operating conditions. Furthermore, the materials must largely accommodate current plant practices and operations, economic considerations, and impacts on the fuel cycle [4]. Related to the latter is the potential for increased releases of tritium from $\mathrm{FeCrAl}$ fuel cladding compared with Zr-based cladding. This paper 
quantifies the kinetics of hydrogen permeation and discusses the prospects, impacts, and potential mitigation strategies for the release of hydrogen isotopes from $\mathrm{FeCrAl}$ cladding.

Tritium is produced in current LWRs via a multitude of pathways, but the reactor inventory is dominated by ternary fission in fuel rods, with a yield of $8 \times 10^{-5}$ atoms/fission (> 12,000 Ci per year for a typical $1000 \mathrm{MWe}$ plant), and neutron absorption by boron $\left({ }^{10} \mathrm{~B}(\mathrm{n}, 2 \alpha)^{3} \mathrm{H}\right)$ in pressurized water reactors (PWRs). In boiling water reactors (BWRs), the only significant source of tritium is the fuel, because of the absence of soluble boron in the primary coolant. Once in the reactor coolant, tritium can undergo isotope exchange reactions with hydrogen-containing chemicals (e.g., $\mathrm{H}_{2} \mathrm{O}, \mathrm{H}_{2}$ ) because tritium behaves chemically identical to hydrogen. HTO molecules are inseparable from water by chemical means, and the proper disposal of tritiumcontaminated water is costly. Additionally, a coolant leak or vapor venting from the core could potentially expose plant personnel to radiation. The continuous production of tritium in the reactor core, combined with its long half-life $\left(t_{1 / 2}=12.33\right.$ years $)$, will maintain tritium activity in an operating reactor. While tritium radiation ( $\beta$ decay) can be shielded, a high level of tritium release is clearly undesirable [5].

The high mobility of tritium allows it to permeat the cladding and enter the primary coolant. The extent of the permeation depends on clad type. Data on the tritium permeation fraction in Zircaloy cladding as compared to from the conventional austenitic stainless steel shows a large difference [5]. Permeation of tritium through Zr-based alloys is roughly 2 orders of magnitude lower than through stainless steel because of the strong 
chemical affinity of zirconium for hydrogen [6-8] and the extremely low tritium permeability of the zirconium oxide that forms on the cladding surface under normal reactor operating conditions [9-11]. Operational experience with several nuclear power plants in the 1970s shows that tritium releases to the environment from PWR and BWR plants using austenitic stainless steel-clad fuel were significantly higher than from plants with Zircaloy-clad fuel assemblies [5].

Any increase in tritium release from the fuel will be directly proportional to the increase in the total tritium inventory in the primary coolant and the potential tritium release to the environment, and this is directly related to the cladding choice. Note that hydrogen permeability of BCC iron alloys is known to be higher than the permeability of facecentered-cubic (FCC) variants [12], given that the BCC lattice is less tightly packed. Thus, it can be conjectured that with FeCrAl fuel cladding, the tritium level in the primary coolant will be even higher than for nuclear power plants that have historically used an austenitic stainless steel fuel clad; of course, it will also be higher than current LWRs, and this raises safety concerns. Therefore, this study conducted a detailed investigation of tritium permeation in different $\mathrm{FeCrAl}$ alloys for accident-tolerant fuel cladding.

The behavior of hydrogen isotopes in austenitic [13-21] and ferritic-martensitic steels $[12,22-27]$ has been studied both experimentally and theoretically at considerable depth since the 1960s. These studies aimed to measure hydrogen isotope permeability, diffusivity, and solubility and to investigate the rate-limiting mechanisms of hydrogen 
isotope transport through these alloys. In the present research, the hydrogen permeability of several $\mathrm{FeCrAl}$ alloys was measured by applying a static pressure change method [28]; this method is different from the commonly used gas evolution permeation technique [26, 29] and is simpler, more straightforward, and less expensive. In addition, a 1-dimensional steady state diffusion model has been developed to describe the tritium pathway from the fuel to primary coolant, and to predict the total tritium inventory within the coolant of light water reactors using FeCrAl-clad fuel assemblies. The present work is expected to enrich the current database for hydrogen isotope permeation in metals and to complement overall R\&D efforts to develop FeCrAl alloys as candidate accident-tolerant fuel cladding materials [1].

\section{Experimental Procedure}

A schematic diagram of the permeation apparatus used in this work is shown in Fig. 1. The physical principle of this technique involves recording the pressure evolution inside a welded cladding segment due to the hydrogen flux through the clad wall of the cladding to a high-vacuum region outside of it.

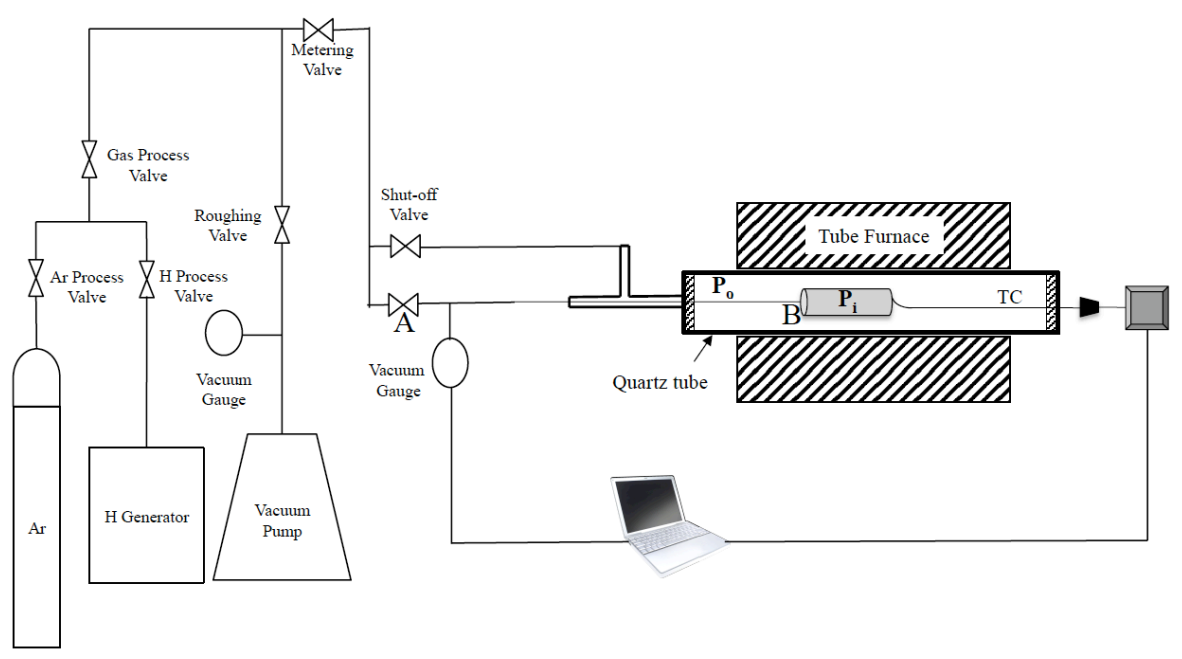


Fig. 1 Schematic view of the permeation apparatus. A-B represents the feeding tube welded to the sample cladding. $\mathrm{P}_{\mathrm{i}}$ and $\mathrm{P}_{\mathrm{o}}$ are hydrogen pressures inside and outside the sample cladding. TC indicates the thermocouple location.

The chemical compositions and dimensions of the materials investigated are listed in Table 1. The T35Y2 and T54Y2 FeCrAl alloys were manufactured (via arc melting, casting, and rolling), machined, and laser-welded at Oak Ridge National Laboratory (ORNL). Detailed microstructure characterization of this nuclear grade wrought FeCrAl alloy can be found in Refs. [30, 31]. The major features are summarized here briefly. The as-received microstructures demonstrated numerous dislocations organized in a pronounced cell structure, as expected from the cold worked condition of these samples. The line dislocation density in the alloys is on the order of $10^{14} \mathrm{~m}^{-2}$. The grain sizes are in a range of 20 60 $\mu \mathrm{m}$. Commercial 304SS and APMT were machined and welded at ORNL. Claddings made of FeCrAl alloy and 304SS were welded to a small 304SS feeding tube (area A-B in Fig. 1) that has dimensions of $0.35 \mathrm{~m}$ in length, $0.0016 \mathrm{~m}$ in diameter, and $0.0005 \mathrm{~m}$ in thickness. The sample assembly was designed with a minimal volume to achieve accurate pressure-change measurements. After attachment of the controlling parts connecting to a gas/vacuum management system, this sample assembly was placed inside a quartz tube $(0.56 \mathrm{~m}$ length, $0.019 \mathrm{~m}$ diameter, and $0.002 \mathrm{~m}$ thickness), which was then inserted into a single-zone horizontal tube furnace capable of heating to a temperature of $1100^{\circ} \mathrm{C}$ with multiple temperature segments. The real temperature of the sample cladding was measured by a K-type thermocouple inserted into the quartz tube through the other end. A tight contact between the thermocouple tip and the bottom of the sample cladding allows accurate temperature determination. The 
gas/vacuum management system was the same as that used in the static hydriding system discussed in $\mathrm{Hu}$ et al. [32]. Ultra-high-purity argon was used to purge the quartz tube multiple times prior to reaching the desired vacuum. A high vacuum $\left(10^{-4}\right.$ torr $)$ was maintained during the measurements to avoid potential oxidation of the sample surface. Any trace oxygen in the quartz tube was further captured by using coarse zirconium metal particles as an oxygen getter inside the tube.

Hydrogen, instead of tritium, was used in this study, based on safety and availability considerations. The isotope effect will be discussed briefly in Section 3.2. Hydrogen with a purity of $99.9995 \%$ was supplied by a VWR ${ }^{\circledR}$ hydrogen generator, in which hydrogen gas is produced by electrolytic dissociation of deionized water before passing through a desiccant cartridge. The gas generator is capable of producing a constant stream of hydrogen with a maximum gas flow rate of $260 \mathrm{cc} / \mathrm{min}$ at $0.13--0.79 \mathrm{MPa}$. After the sample cladding was purged three times with high-purity argon gas, high-purity hydrogen was introduced into the cladding via a metering valve to reach the desired pressure value. Throughout a pre-set temperature profile, the hydrogen pressure inside the cladding was recorded.

Table 1. Chemical compositions (wt \%) and dimensions of the alloys used for permeation tests

\begin{tabular}{ccccccccccccc}
\hline \multirow{2}{*}{ Materials } & \multicolumn{1}{c}{ Chemical compositions (wt \%) } & & & & Dimensions (m) \\
\cline { 2 - 12 } & Fe & Cr & Al & Y & Mo & Mn & C & S & Si & Length diameter wall thickness \\
\hline $304 \mathrm{SS}$ & Bal. & 18.35 & - & - & 0.14 & 1.31 & 0.062 & 0.02 & 0.57 & 0.051 & 0.0127 & 0.00051
\end{tabular}




$\begin{array}{lcccccccccccc}\text { T35Y2 } & \text { Bal. } & 13.15 & 4.44 & 0.12 & - & <0.01 & 0.002 & 0.0003 & 0.01 & & & \\ \text { T54Y2 } & \text { Bal. } & 15.06 & 3.93 & 0.12 & - & <0.01 & 0.002 & 0.0003 & 0.02 & & & \\ \text { APMT } & \text { Bal. } & 21 & 5 & - & 3 & <0.4 & 0.05 & - & <0.7 & 0.0254 & 0.0095 & 0.00051\end{array}$

Assuming 1-dimensional transport, the flux, $J$, of hydrogen permeating through the sample clad can be derived from Fick's first law, assuming hydrogen solubility governed by Sieverts' law, and is given by

$$
J=\frac{D S\left(P_{i}^{1 / 2}-P_{o}^{1 / 2}\right)}{\delta}
$$

where $\delta$ is the clad wall thickness of the sample, and $P_{i}$ and $P_{o}$ are the hydrogen pressures inside and outside the sample cladding, respectively, as shown in Fig. 1. $P_{o}$ is negligible because the quartz tube is always under a high vacuum during measurement. The product of diffusivity, $D$, and Sievert's parameter, $S$, (note that $S$ is usually defined as "solubility" in hydrogen permeation studies [21]) is defined as the permeability, $\Phi$, which can be expressed in the form of

$$
\phi=\frac{J \delta}{P_{i}^{1 / 2}}
$$

Units used here for $D$ and $S$ are, respectively, $\mathrm{m}^{2} / \mathrm{s}$ and $m o l H_{2} / m^{3} / \sqrt{M P a}$, giving rise to the unit of $\Phi, \mathrm{molH}_{2} / \mathrm{m} / \mathrm{s} / \sqrt{M P a}$. 
The pressure changes inside the sample cladding were continuously recorded; these changes can be used to obtain the hydrogen flux through the cladding surface, with a known temperature and sample volume and area.

For most metals and alloys, permeability follows an Arrhenius temperature dependence. Thus it can be expressed as

$$
\phi=\phi_{0} \exp \left(-\frac{E}{R T}\right)
$$

where $\Phi_{0}$ is the permeability constant and $E$ is an apparent activation energy for permeation [21] (essentially the sum of activation energies for dissolution and diffusion).

\section{Experimental results}

\subsection{System calibration and validation}

For the purposes of this study, it was important to have a constant, uniform temperature along the sample cladding to produce homogeneous hydrogen flux throughout the sample cladding surface. The temperature profile was not flat across the whole heating zone in the tube furnace, as shown in Fig. 2; the data in Fig. 2 were obtained by inserting a thermocouple at different positions inside the furnace after it reached steady state. The sample cladding was placed in the center of the heating zone, inside a uniform temperature region, as shown in Fig. 2. Several temperature segments were set during each measurement, and pressure changes under steady state temperature regimes were 
extracted for data analysis. A typical temperature profile and the corresponding pressure changes are shown in Fig. 3.

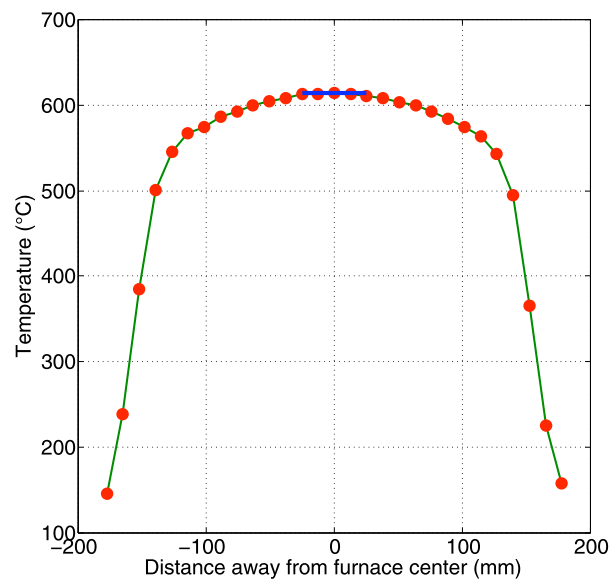

Fig. 2 Temperature profile of the tube furnace heating zone when the furnace temperature is set at $600^{\circ} \mathrm{C}$.

The blue line demonstrates the position of the sample cladding.

The measured pressure change was due to the hydrogen permeation through the sample cladding, as well as through the feeding tube (A-B), which complicates the interpretation of the pressure change results. To acquire an accurate estimation of the hydrogen flux from the sample cladding only, hydrogen leakage from the feeding tube must be subtracted. Therefore, the feeding tube (A-B) with a weld-sealed end (B) was placed in the quartz tube to measure hydrogen permeation for calibration by setting a temperature profile identical to that shown in Fig. 3. Note that this tube was located in exactly the same position as it would be if it were welded to the sample claddings. It is obvious that a significant temperature gradient exits across the length of the feeding tube; and the hot region is $\sim 0.15 \mathrm{~m}$, from which the majority of the hydrogen is believed to be released. However, the hydrogen permeation rate of this feeding tube could not be directly 
subtracted from the measured total hydrogen flux of a complete sample assembly, because the hydrogen flux is a function of pressure, which varies across the different measurements. Therefore, an equivalent hydrogen permeability of the feeding tube was necessary to compute hydrogen flux at different conditions.

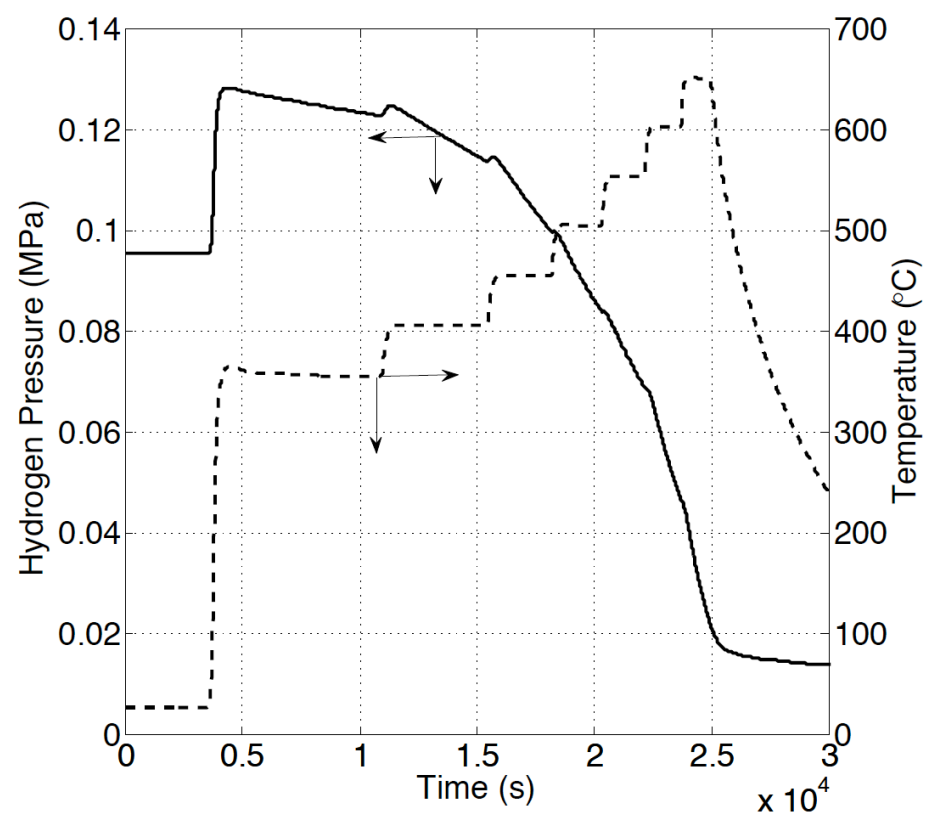

Fig. 3 Temperature profile and hydrogen pressure evolution during a typical permeation test.

To simplify the calculation, an equivalent $0.12 \mathrm{~m}$ long section of the feeding tube, at fixed temperatures consistent with thermocouple readings, was assumed to transmit hydrogen. Three calibrations were performed and are shown in Fig. 4, along with the fitting curve. The resulting "effective hydrogen permeability" of this feeding tube is expressed as 


$$
\phi_{\text {Feeding Thbe }}\left[\mathrm{mol} \mathrm{H} \mathrm{m}^{-1} \mathrm{~s}^{-1} \mathrm{MPa}^{-1 / 2}\right]=(2.44 \pm 2.05) \times 10^{-3} \exp \left(\frac{73.4 \pm 3.9\left[\mathrm{ES} \mathrm{mol}^{-1}\right]}{R T}\right) \text {. }
$$

This correlation was used in Eq. (2) to subtract the hydrogen loss from the feeding tube. After this correction, the data were then used to calculate the net hydrogen flux from the cladding section only. This methodology implies that the accuracy of the system (lowest detection limit) is limited by the hydrogen flux from the feeding tube.

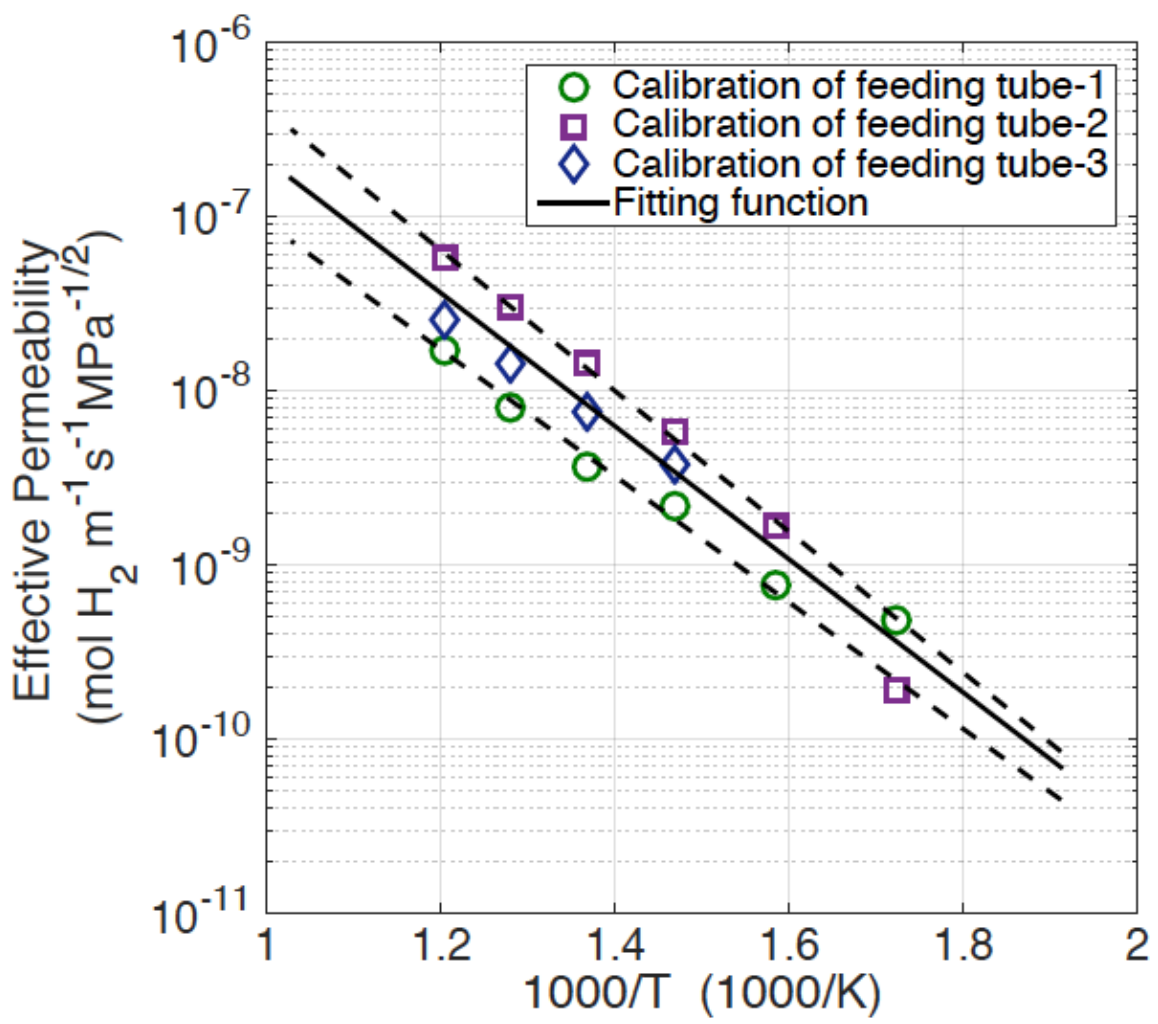

Fig. 4 Equivalent hydrogen permeability of the feeding tube. The solid line is the fitting result expressed in Eq. (4) and the two dashed lines label the uncertainties. 
304SS cladding was used initially to validate the experimental system and analysis approach because hydrogen permeability has been studied extensively for this material. Figure 5 shows the hydrogen permeability determined from 304SS using the system described here, along with select literature data. The measured hydrogen permeability was slightly lower than, though in adequate agreement with, what is reported in the literature $[15,17,19,27]$. The repeatability of the measurements was also shown to be adequate.

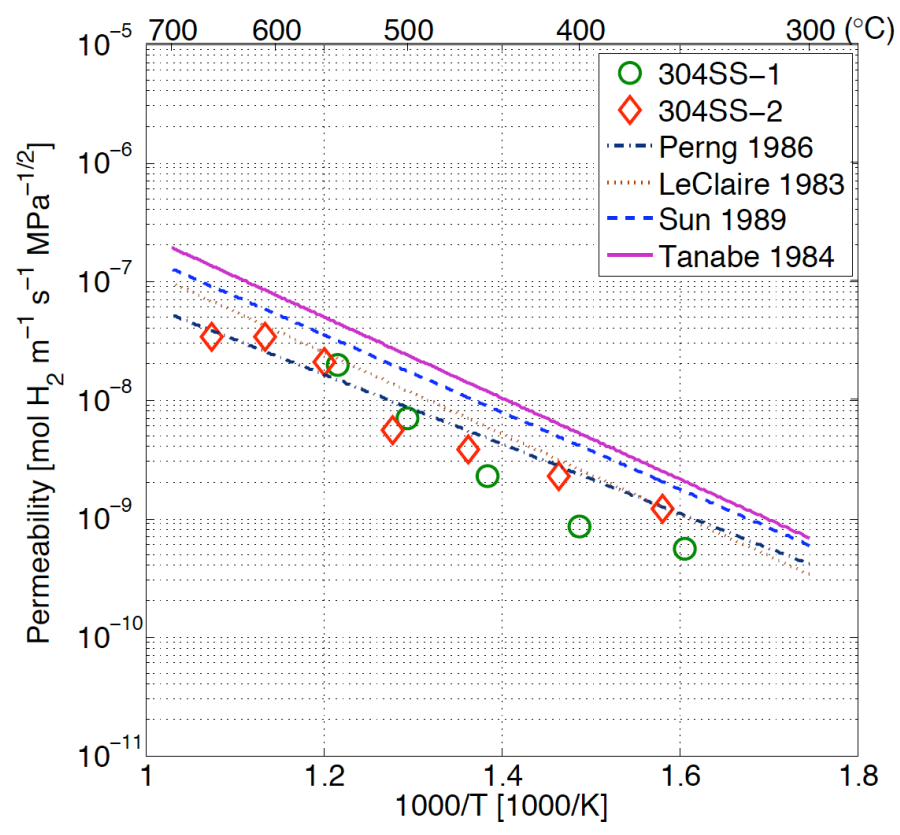

Fig. 5 Comparison of hydrogen permeability of 304SS cladding, measured using the present experimental technique, and literature data

\subsection{Hydrogen permeability of FeCrAl alloys}

The hydrogen permeability of three different FeCrAl alloys was determined at various temperatures between 350 and $650^{\circ} \mathrm{C}$ and are plotted in Fig. 6. To obtain a direct 
comparison, the permeability data for 304SS measured using the same system are also shown. It appears that the hydrogen permeability of FeCrAl alloys is five times greater than that of $304 \mathrm{SS}$ at $350^{\circ} \mathrm{C}$ and three times higher at $650^{\circ} \mathrm{C}$ because of the difference in crystal structrue. Compared with pure iron (brown dashed line) [26], the hydrogen permeabilities of the FeCrAl alloys are one to two orders of magnitude lower, which is consistent with the results reported in Van Deventer and Maroni [22]. The black dashed line in Fig. 6 indicates the hydrogen permeability of 406SS (Fe-13Cr-3.9Al), reported in Ref. [14], which is very close to that of T35Y2 and T54Y2; the similarity in permeability is attributed to their similar compostition. APMT with higher $\mathrm{Cr}$ and $\mathrm{Al}$ contents has smaller hydrogen permeability in comparison with T35Y2 and T54Y2. Considering the potential application of these materials as fuel claddings, the hydrogen permeabilities of Zircaloy-2 and Zircaloy-4 - the current fuel cladding materials for BWRs and PWRs, respectively — are also plotted in the same figure. As expected, the results indicate that hydrogen permeation in the FeCrAl alloys is nearly 2 orders of magnitude higher. Therefore, a larger tritium inventory in the primary coolant is to be expected for reactors using FeCrAl-clad fuel assemblies. Furthermore, it is known that tritium permeation through Zr-alloy cladding is reduced dramatically once zirconium oxide (an effective tritium barrier) forms on the innner and outer clad surfaces. 


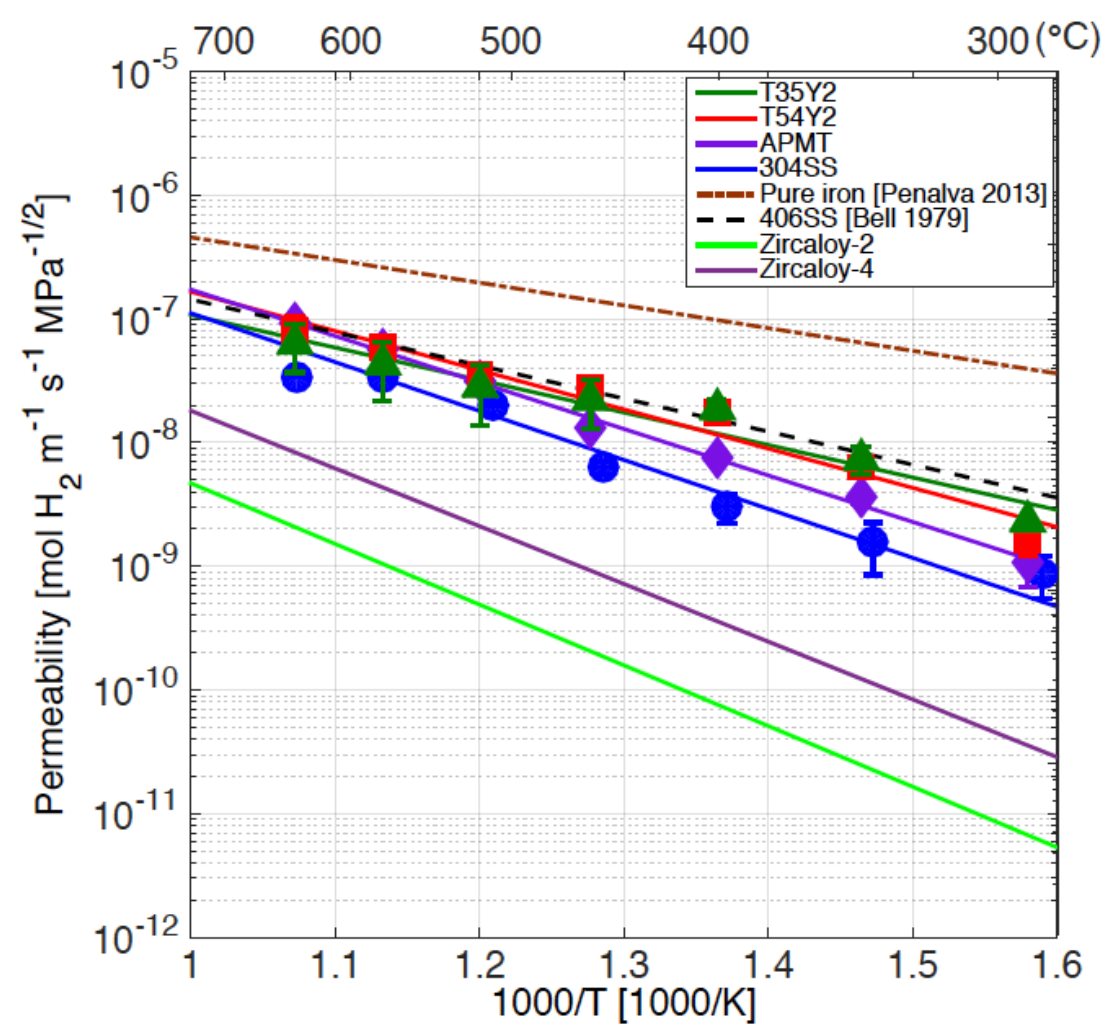

Fig. 6 Arrhenius plot of the fitted hydrogen permeability for T35Y2, T54Y2, APMT, 304SS, 406SS, Zircaloy-2, Zircaloy-4, and pure iron over a 350 to $650{ }^{\circ} \mathrm{C}$ range. The permeability of Zircaloy- 2 and Zircaloy-4 was computed using the data in Refs. [33] and [34].

The experimentally obtained permeation data were fitted using a nonlinear least-squares method. For the samples analyzed, the permeability did indeed show an Arrhenius type dependence on temperature. These relationships are graphically shown in Fig. 6 and correspond to the following analytical expressions: 
$\phi_{H, T 35 Y 2}\left[\mathrm{~mol} \mathrm{H} \mathrm{m}^{-1} \mathrm{~s}^{-1} \mathrm{MPa}^{-1 / 2}\right]=(4.49 \pm 2.35) \times 10^{-5} \exp \left(-\frac{50.2 \pm 5.96\left[\mathrm{KJ} \mathrm{mol}^{-1}\right]}{R T}\right)$

$\phi_{H, T 54 Y 2}\left[\operatorname{mol~H} \mathrm{H}_{2} \mathrm{~m}^{-1} \mathrm{sPa}^{-1 / 2}\right]=(2.43 \pm 0.64) \times 10^{-4} \exp \left(-\frac{60.6 \pm 0.6\left[\mathrm{rJmol} \mathrm{I}^{-1}\right]}{R T}\right)$

$\phi_{H, A P M T}\left[\mathrm{~mol} \mathrm{H} \mathrm{m}^{-1} \mathrm{~s}^{-1} \mathrm{MPa}^{-1 / 2}\right]=(9.77 \pm 2.23) \times 10^{-4} \exp \left(\frac{71.8 \pm 5.8\left[\mathrm{E} J \mathrm{~mol}^{-1}\right]}{R T}\right)$

Jung [24] showed that the activation energy of hydrogen permeation increases with increasing chromium content, which is in agreement with the trend of the activation energies provided in Eqs. (5-7). It is emphasized here that microstructure effect is not discussed and the permeability shown in Eqs. (5-7) corresponds only to apparent measured data in accordance with literature definition of permeability.

The tritium permeability in these $\mathrm{FeCrAl}$ alloys can be determined from the ratio of permeability between hydrogen and tritium that is equivalent to the inverse ratio of the square root of the masses of the isotopes [21]:

$$
\phi_{H}=\sqrt{2} \phi_{D}=\sqrt{3} \phi_{T} .
$$

\section{Discussion}

\subsection{One-dimensional steady state tritium diffusion model}


This section introduces a 1-dimensional steady state tritium diffusion model to demonstrate how the tritium inventory in the primary coolant of a typical $1000 \mathrm{MWe}$ four-loop PWR and a traditional 1000 MWe BWR would change if FeCrAl alloys were used as fuel clad.

\subsubsection{Model description}

\section{$\underline{\text { Tritium in fuel }}$}

Tritium generated in fuel rods is released into the primary coolant via four stages: transport within the fuel pellets, entrance into the fuel-clad gap, absorption by the cladding internal surface, and diffusion through the cladding and its protective oxide film to reach the coolant (Fig.7). Note that most of the tritium formed within $\mathrm{UO}_{2}$ pellets remains in the fuel. The amount of tritium retained in the fuel pellets, which is formed in ternary fission, is a function of fuel linear power and burnup. At higher linear powers, with corresponding higher fuel temperatures, a greater fraction of the tritium is released from the fuel pellets into the internal volume of the fuel pin. For example, Dolle et al. [35] showed that at $35,000 \mathrm{MWd} / \mathrm{MTU}$ burnup, a linear power of $137 \mathrm{~W} / \mathrm{cm}$, and a corresponding volumetric average temperature of $650^{\circ} \mathrm{C}, 80 \%$ of the tritium is retained in the fuel. At a volumetric average temperature of $850^{\circ} \mathrm{C}(220 \mathrm{~W} / \mathrm{cm})$, this number is reduced to $50 \%$. Tritium retention in the fuel is likely aided by the formation of metal hydrides (tritides) in the outer portion of the pellets and the formation of $\mathrm{T}_{2} \mathrm{O}$ due to the increasing oxygen potential. The relatively large fission yield of elements such as 
zirconium, rare earths, and barium, which readily form hydrides, are available to react with the smaller quantities of tritium formed [5]. As power and corresponding fuel temperatures increase, it is anticipated that increased rates of metal tritide decomposition and more rapid tritium diffusion produce an increase in the amount of tritium released into the fuel pin internal volume.

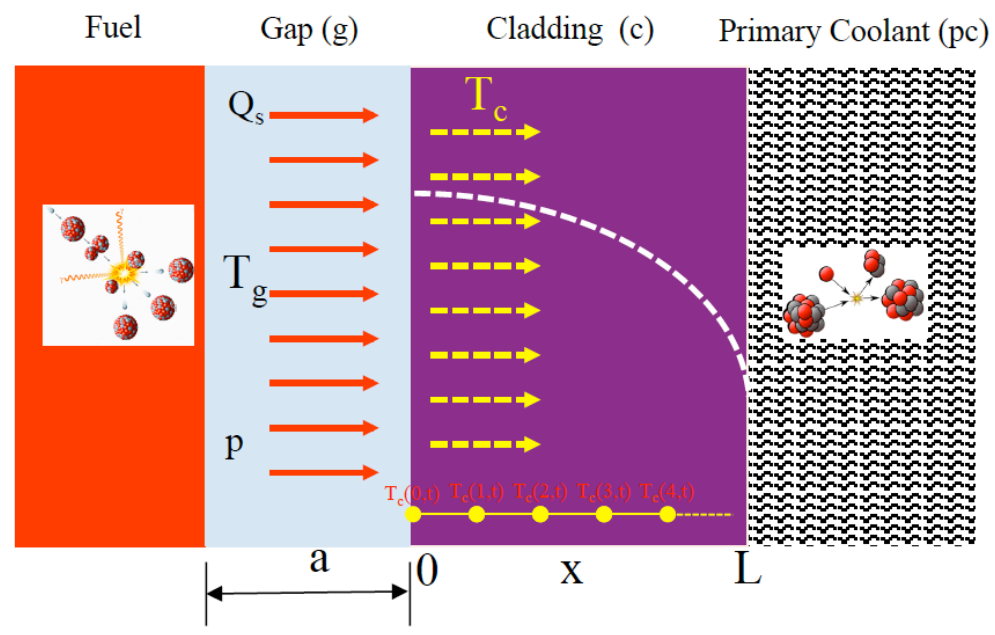

Fig. 7 Tritium pathway from fuel pellet to primary coolant. $Q_{s}$ is the hypothetical homogeneous tritium source released from fuel. $\mathrm{T}_{\mathrm{g}}$ indicates the tritium concentration in the fuel-cladding gap. $\mathrm{p}$ is the tritium partial pressure in the fuel-cladding gap. a refers to the fuel-cladding gap width. $\mathrm{T}_{\mathrm{c}}$ is the tritium concentration in the cladding, a function of time and position. $\mathrm{L}$ is the thickness of the cladding. $\mathrm{x}$ indicates the distance away from the cladding internal surface. The line segments demonstrate the discretization process for numerical solution of the tritium concentration in cladding. More details about these symbols can be found in the text.

The tritium production rate in the fuel is based on the operating conditions. Assuming that a fuel rod is operating at $220 \mathrm{~W} / \mathrm{cm}$, then an $8.2 \mathrm{~mm}$ diameter fuel pellet (LWR $17 \times 17$ geometry) will have a power density of $416.6 \mathrm{~W} / \mathrm{cm}^{3}$. Therefore, the fission rate is 
$1.26 \times 10^{13}$ fissions $/ \mathrm{cm}^{3} / \mathrm{s}$, assuming $200 \mathrm{MeV} /$ fission and this produces a cumulative tritium yield of $1.18 \times 10^{-4}$ [36]. Therefore, the tritium production rate in the fuel is estimated to be $1.335 \times 10^{9}$ atoms $/ \mathrm{cm}^{3} \cdot \mathrm{s}$. As suggested in Ref. [35], $50 \%$ of the tritium produced in the fuel is assumed to be released into the fuel cladding gap immediately after it is generated in the fuel, leading to a hypothetical homogeneous tritium source term in the gap, $Q_{s}$.

\section{Tritium in fuel-cladding gap}

Tritium inside the gap can be absorbed onto the inner clad surface of the cladding, which is governed by Sievert's law. The time-dependent evolution of the tritium concentration inside the fuel-clad gap, $T_{g}(t)$ with a unit of $\mathrm{cm}^{-3}$, can be expressed as

$$
\frac{d T_{g}(t)}{d t}=Q_{s}-Q_{g-c}-\lambda T_{g}(t)
$$

where $Q_{g-c}$ is the rate of absorption of tritium by the clad internal surface and the last term on the right hand side of the equation refers to the annihilation rate of tritium due to decay; $\lambda$ is the decay constant of tritium $\left(\lambda=\frac{\ln 2}{t_{1 / 2}}\right)$.

\section{$\underline{\text { Tritium in cladding }}$}


Local equilibrium is assumed at the inner gap-cladding interface, implying that the tritium cannot accumulate on this surface, and the flux from the gap into the cladding surface is equivalent to that from the surface into the cladding bulk:

$$
J_{g-i}=J_{i-c}
$$

Since a homogeneous tritium distribution is assumed in the gap, combing Eqs. (9) and (10) provides an expression of the tritium flux from the gap into the cladding as

$$
J_{g-i}=a Q_{g-c}=a\left(\frac{d T_{g}(t)}{d t}-Q_{s}+\lambda T_{g}(t)\right),
$$

where $a$ is the ratio of the gap volume and the inner surface area of the cladding, with a unit of length in $\mathrm{m}$.

The tritium transport in FeCrAl cladding is diffusion controlled. The tritium flux from the cladding inner surface into the bulk is described by Fick's first law,

$$
J_{i-c}=-D_{T} \frac{\partial T_{c}(0, t)}{\partial x}
$$

where $D_{T}$ is the tritium diffusivity in $\mathrm{FeCrAl}$ alloys and is assumed to be constant and independent of temperature and concentration (Note that Soret effect is not considered in this model); $T_{c}(0, t)$ is tritium concentration at the cladding internal surface; and $x$ is the distance away from gap-cladding interface. 
Based on Sievert's law, the tritium concentration is proportional to $p^{1 / 2}$, where $p$ is the tritium partial pressure inside the gap. Subsequently, the expression of equilibrium tritium concentration at the cladding internal surface is

$$
T_{c}(0, t)=S p^{1 / 2}=S \alpha^{\prime} T_{g}^{1 / 2}
$$

where $S$ is the tritium solubility in the clad materials; $\alpha^{\prime}$ is the coefficient used to transform the tritium concentration to gas pressure in the gap, derived from the ideal gas state equation.

Combing Eqs. (10-13) leads to the first boundary condition for the partial differential equation used to describe the tritium evolution inside the cladding, in the form of

$$
2 \alpha a T_{c}(0, t) \frac{\partial T_{c}(0, t)}{\partial t}-a Q_{s}+\alpha \lambda a\left(T_{c}(0, t)\right)^{2}=D_{T} \frac{\partial T_{c}(0, t)}{\partial x}
$$

where $\alpha=\frac{1}{S^{2} \alpha^{\prime 2}}$

The evolution of tritium inside the cladding is described by Fick's second law,

$$
\frac{\partial T_{c}(x, t)}{\partial t}=D_{T} \frac{\partial^{2} T_{c}(x, t)}{\partial x^{2}}-\lambda T_{c}(x, t)
$$


The initial condition of Eq. (15) is $T_{c}(x, 0)=0$. Besides the boundary condtion of Eq. (14) at the gap-cladding interface, a second boundary condition is needed to solve Eq. (15).

At the waterside interface of the cladding and the primary coolant, at a distance $L$ away from the gap-cladding interface that is also equal to the cladding thickness, we assume that tritium experiences an instantaneous isotope exchange to form HTO [37] once it reaches this surface, implying the second boundary condtion,

$$
T_{c}(L, t)=0
$$

\section{$\underline{\text { Tritium in primary coolant }}$}

Tritium from ternary fission is only one source of the tritium inventory in the primary coolant of PWRs. The other significant contribution comes from neutron absorption in soluble boron, following two major pathways-

$$
\begin{aligned}
& { }^{10} B+n \rightarrow{ }^{7} \mathrm{Li}+\alpha \quad 3837 b \text { for thermal } n \\
& { }^{7} \mathrm{Li}+n \rightarrow n+\alpha+{ }_{1}^{3} \mathrm{H} \quad 330 \mathrm{mb} \text { for fast } n(E>2.5 \mathrm{MeV}) \\
& { }^{10} \mathrm{~B}+n \rightarrow{ }^{8} \mathrm{Be}+{ }_{1}^{3} \mathrm{H} \quad 42 \mathrm{mb} \text { for fast } n(\mathrm{E}>0.1 \mathrm{MeV}) .
\end{aligned}
$$

The boron concentration in the primary coolant has been assumed to linearly decrease from $\sim 1200$ ppm to zero throughout each operation cycle,

$$
N_{B}=k_{1}-k_{2} t \text {, }
$$


where $\mathrm{k}_{1}=1200 \mathrm{ppm}, \mathrm{k}_{2}=6.67 \mathrm{ppm} / \mathrm{month}$.

For BWRs, these reactions are not considered because of the absence of soluble boron in the coolant; this indicates that the only significant tritium source in BWR primary coolant will be ternary fissions in the fuel.

The amount of tritium generated by reaction (17) is derived from the following two equations,

$$
\begin{gathered}
\frac{d N_{L i}}{d t}=N_{B} \phi_{t n} \sigma_{1}-N_{L i} \phi_{f n} \sigma_{2}, \\
\frac{d T_{1}}{d t}=N_{L l} \phi_{f n} \sigma_{2}-\lambda T_{1} .
\end{gathered}
$$

where $N_{L i}$ is lithium concentration in the primary coolant, $N_{B}$ is boron concentration in the primary coolant, $T_{1}$ is the tritium concentration produced by reaction $(17), \phi_{t n}$ is average thermal neutron flux in the reactor, $\phi_{f n}$ is average fast neutron flux in the reactor, and $\sigma$ is the neutron absorptioncross section, where $\sigma_{1}=3837 \mathrm{~b}$ and $\sigma_{2}=330 \mathrm{mb}$. Combing equations (19), (20), and (21) leads to the solution of $\mathrm{T}_{1}$,

$$
T_{1}=\frac{p}{\lambda}+\frac{k_{2} \phi_{t n} \sigma_{1}}{\lambda^{2}}+\frac{p}{\phi_{f n} \sigma_{2}-\lambda} \exp \left(-\phi_{f n} \sigma_{2} t\right)-\frac{k_{2} \phi_{t n} \sigma_{1}}{\lambda} t+q \exp (-\lambda t)
$$

where $p=k_{1} \phi_{t n} \sigma_{1}+\frac{k_{2} \phi_{t n} \sigma_{1}}{\phi_{f n} \sigma_{2}} ; q=-\left(\frac{p}{\phi_{f n} \sigma_{2}-\lambda}+\frac{p}{\lambda}+\frac{k_{2} \phi_{t n} \sigma_{1}}{\lambda^{2}}\right)$.

Additionally, tritium $\left(\mathrm{T}_{2}\right)$ also could be generated by reaction (18) in the form of

$$
\frac{d T_{2}}{d t}=N_{B} \phi_{f n} \sigma_{3}-\lambda T_{2}
$$

where $\sigma_{3}$ is the cross section for (n, B) reaction expressed in (18). 
The analytical solution of this equation is

$$
T_{2}=\phi_{f n} \sigma_{3} \frac{\lambda k_{1}+k_{2}}{\lambda^{2}}[-\exp (-\lambda t)]-\frac{k_{2} \phi_{f n} \sigma_{3}}{\lambda} t
$$

Therefore, the total tritium inventory in the primary coolant of PWRs is the superposition of (21), (23), and the diffusion flux from the cladding into the coolant.

\subsubsection{Numerical solution}

The most critical part of this problem is the tritium distribution across the cladding, described in Eq. (15), which connects the tritium stored in the fuel-cladding gap and the primary coolant by using Eq. (14) as boundary condition and by providing input for Eq. (19).

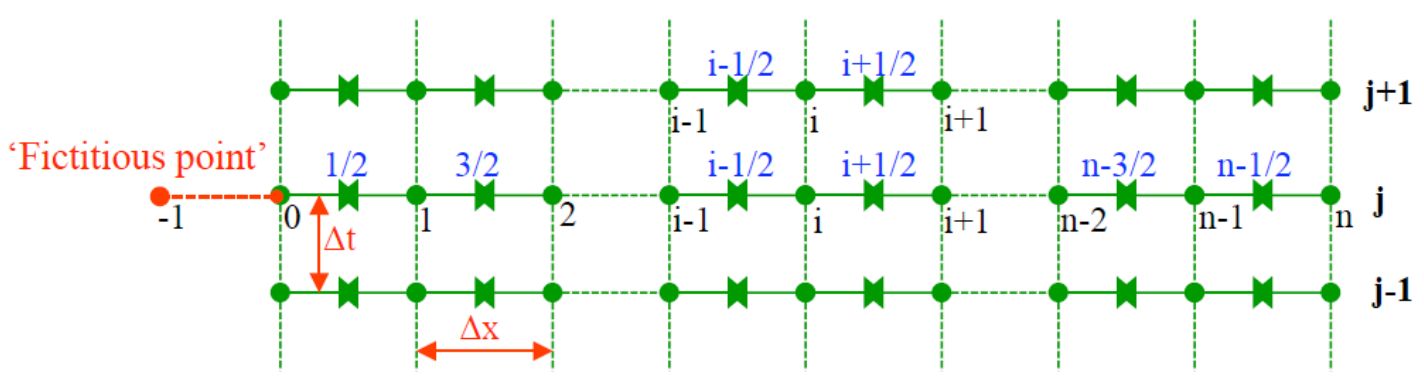

Fig. 8 Crank-Nicolson discretization scheme.

A finite-difference method using a semi-implicit Crank-Nicolson [38] scheme was implemented to solve Eq. (15), as shown in Fig. 8. The discretized form of Eq. (15) using this scheme is 


$$
\begin{aligned}
& \frac{T_{c, i}^{j+1}-T_{c, i}^{j}}{\Delta t}=\frac{D_{T}}{2}\left(\frac{\partial^{2} T_{c, i}^{j+1}}{\partial x^{2}}+\frac{\partial^{2} T_{c, i}^{j}}{\partial x^{2}}\right)-\lambda T_{c, i}^{j+1} \\
& =\frac{D_{T}}{2 \Delta x^{2}}\left(T_{c, i+1}^{j+1}-2 T_{c, i}^{j+1}+T_{c, i-1}^{j+1}+T_{c, i+1}^{j}-2 T_{c, i}^{j}+T_{c, i-1}^{j}\right)-\lambda T_{c, i}^{j+1} .
\end{aligned}
$$

The boundary condition of Eq. (14) complicates the discretization by introducing the derivation of the first spatial node, for which a fictitious concentration, $T_{c,-1}$ is introduced at the external grid point. Essentially, one should imagine that the cladding was extended by one layer. The explicit form of the first derivative of the tritium concentration at the cladding internal surface is

$$
\frac{\partial T_{c, 0}}{\partial x}=\frac{1}{2 \Delta x}\left(T_{c, 1}-T_{c,-1}\right)
$$

Using Eq. (15), Eq. (21) leads to

$$
\frac{\partial T_{c, 0}}{\partial t}=D_{T} \frac{\partial^{2} T_{c, 0}}{\partial x^{2}}-\lambda T_{c, 0}
$$

where

$$
\frac{\partial^{2} T_{c, 0}}{\partial x^{2}}=\frac{T_{c, 1}-2 T_{c, 0}+T_{c,-1}}{\Delta x^{2}}
$$

Substituting Eqs. (21-23) into Eq. (14) gives rise to the discretized form of this boundary condition as 


$$
T_{c, 0}^{j+1}=\left[1+\frac{1}{2 \alpha a T_{c, 0}^{j}+\frac{\Delta x}{2}}\left(\frac{\Delta t a Q_{s}}{T_{c, 0}^{j}}-\Delta t a \lambda \alpha T_{c, 0}^{j}-\frac{D_{T} \Delta t}{\Delta x}\right)\right] T_{c, 0}^{j}+\frac{D_{T} \Delta t}{\Delta x\left(2 \alpha a T_{c, 0}^{j}+\frac{\Delta x}{2}\right)} T_{c, 1}^{j}
$$

\subsubsection{Results}

Given the methodology introduced, the tritium inventory in the primary coolant can be predicted for both PWRs and BWRs. The kinetics parameters of tritium permeability used in this model is derived based on the experimental data of T54Y2 by applying Eq. (8). Note that the $\mathrm{H}$ permeability of $\mathrm{T} 35 \mathrm{Y} 2$ and $\mathrm{T} 54 \mathrm{Y} 2$ are quite similar and the parameters used in the model are based on that of T35Y2. In order to obtain a direct comparison between the reactors using Zr-alloy fuel clad and $\mathrm{FeCrAl}$ fuel clad, the parameters of a typical 1000 MWe four-loop PWR and a traditional 1000 MWe BWR were applied in this model to predict the total tritium inventory in the primary coolant of the reactors using FeCrAl fuel clad. Tritium evolution across the FeCrAl cladding was examined initially. Figure 9 depicts the contour plots of tritium concentration inside the FeCrAl cladding of a PWR and a BWR. Once these reactors start operating, a steady state value of the tritium concentration is rapidly achieved, given the assumption of instantaneous release of $50 \%$ of the tritium yielded by fission, high mobility of tritium, and a small thickness of FeCrAl cladding. It takes about 33 hours for PWRs and 50 hours for BWRs to reach this equilibrium state, the onset of which implies that the tritium pickup rate by the cladding is equivalent to the releasing rate of tritium into the primary coolant. At this steady state, tritium enters the primary coolant at a constant rate. Note 
that in order to compensate for the neutronic penalty of reactors using FeCrAl cladding, the cladding thickness will most likely be reduced further from that assumed here, which implies an even shorter time to reach equilibrium.

(a)

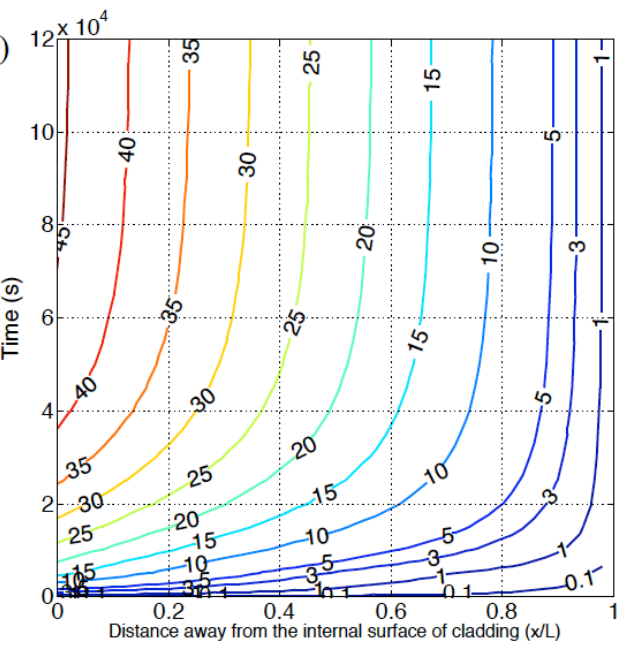

(b)

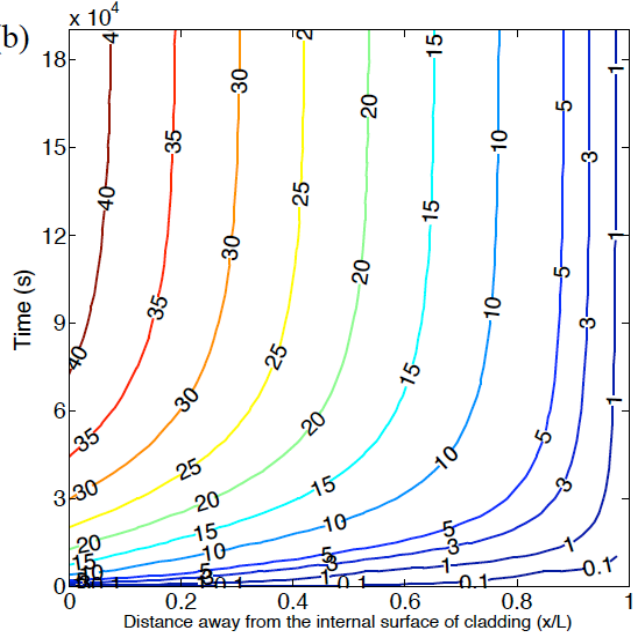

Fig. 9 Tritium evolution across FeCrAl cladding of (a) PWR and (b) BWR. The labeled numbers in the contour plots are $\mathrm{T}_{\mathrm{C}}\left[\mathrm{mol} \mathrm{T} / \mathrm{cm}^{3}\right] \times 10^{12}$.

Figure 10 shows the tritium inventory in the primary coolant of a FeCrAl-clad PWR and BWR during one fuel cycle. As noted earlier, the tritium inventory in a PWR primary coolant is due to contributions from ternary fission in the fuel and from neutron absorption in the coolant. The amount of tritium diffusing from the FeCrAl-clad fuel is comparable to that generated in the coolant. In BWRs, the sole tritium source is that released from the fuel, which is slightly larger than what is predicted for PWRs because of the thinner cladding wall. For current PWRs using Zircaloy as fuel clad, the total tritium inventory in the primary coolant will be given by the amount of tritium produced in the primary coolant, assuming only very little tritium can diffuse from the fuel through 
Zr-alloy clad. This should be even smaller for current BWRs, considering that the sole source of tritium is ternary fission in the fuel pellets. Therefore, the total tritium activity in the primary coolant of a reactor is strongly dependent on the selection of cladding material.

Note that the above calculations are based on the parameters obtained from the measurements on un-irradiated $\mathrm{FeCrAl}$ alloys. It is known that radiation induced microstructure change could modify the mobility and the solubility of tritium in $\mathrm{FeCrAl}$ alloys, leading to a different permeability compared to the un-irradiated condition. The radiation influence on tritium behavior in $\mathrm{FeCrAl}$ is not discussed here and needs further investigations.

(a)

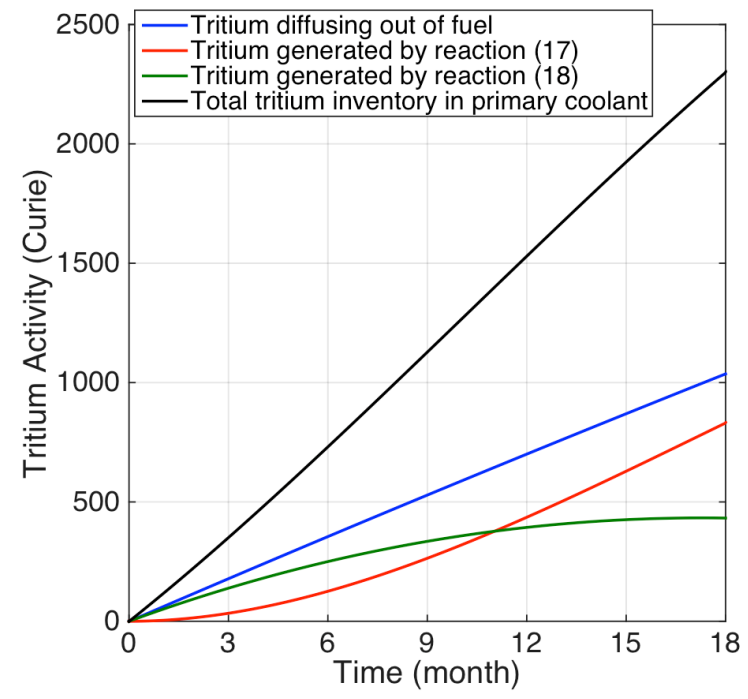

(b)

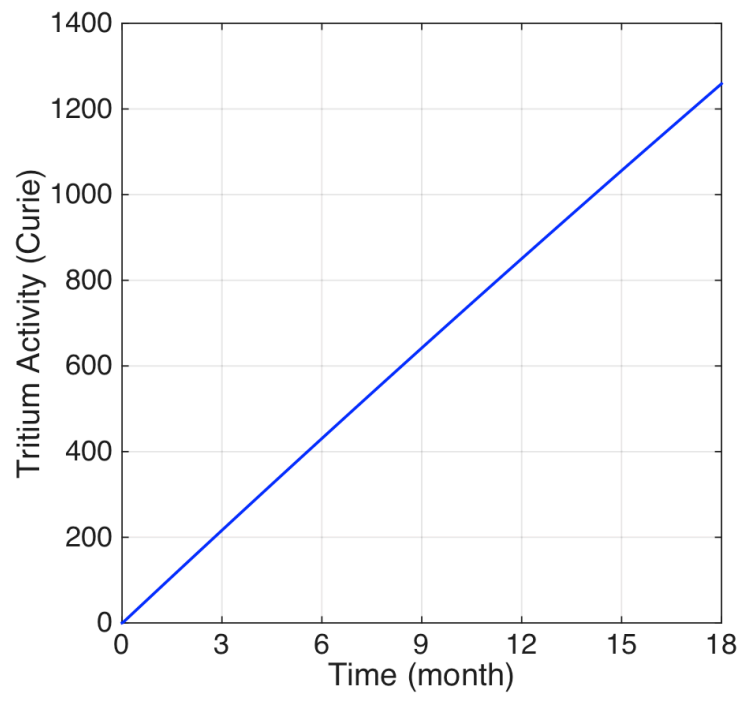

Fig. 10 Tritium activity in primary coolant of (a) PWR and (b) BWR in one fuel cycle operation 


\subsection{Potential tritium mitigation strategy for FeCrAl clad}

In light of the discussion above, it is apparent that the use of FeCrAl alloys as accidenttolerant fuel cladding materials is the potential to dramatically increase the tritium activity in the primary coolant. Although tritium is a relatively weak $\beta$ emitter, the increase in associated dose level is not desirable from an operational standpoint and from ALARA (as low as reasonably achievable) principles. Therefore, a strong incentive exists to reduce the extent of tritium release from the fuel through the clad.
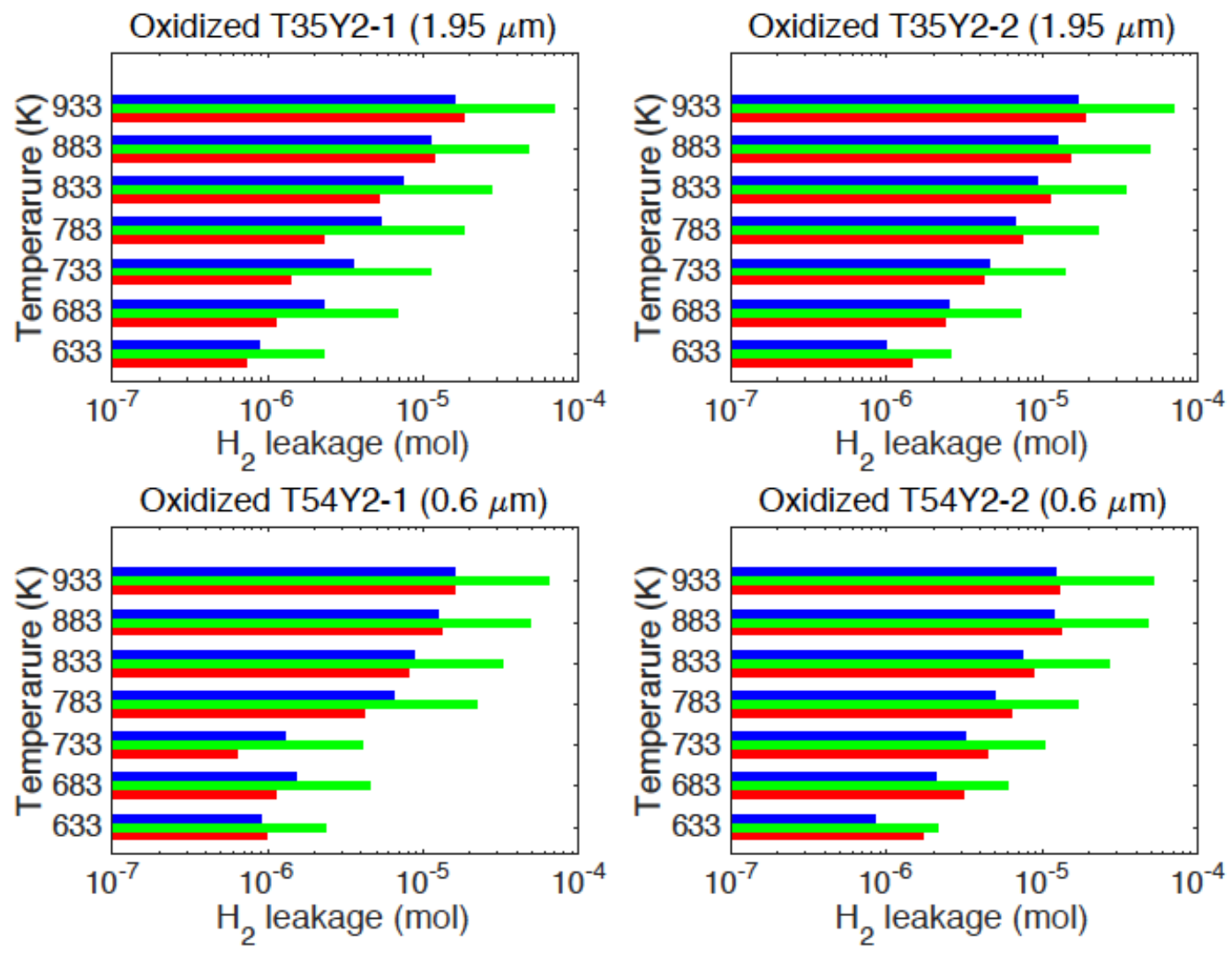
Fig. 11. Hydrogen leakage of oxidized FeCrAl cladding tubes. Red bars refer to measured hydrogen leakage from the system; Green and Blue bars indicate the maximum and minimum of calculated hydrogen leakage from the 304SS feeding tube, respectively.

Externally applied coatings on steel substrates have been examined extensively in the past as tritium barriers, including oxides, aluminides, nitrides, and carbides [11, 39, 40]. The last portion of this study examined the capability of the alumina formed during hightemperature oxidation of $\mathrm{FeCrAl}$ alloys to act as a barrier to tritium transport. In fact, this alumina $\left(\alpha-\mathrm{Al}_{2} \mathrm{O}_{3}\right)$ layer is what enables the excellent oxidation resistance of this class of alloys under severe accident conditions and makes them suitable candidates for accidenttolerant fuel clad applications. $\mathrm{Al}_{2} \mathrm{O}_{3}$ exhibits extremely low tritium permeability, almost ten orders of magnitude lower than that measured in this study for FeCrAl alloys [11].

Due to the limitation of the FeCrAl cladding tubes, a T35Y2 and a T54Y2 sample cladding section were placed in dry air at $1200^{\circ} \mathrm{C}$ for 2 hours and at $1100^{\circ} \mathrm{C}$ for 1.5 hours, respectively. The resulting oxide layers for these two samples were calculated to be $1.9 \mu \mathrm{m}$ and $0.6 \mu \mathrm{m}$, based on the weight gain after oxidation. These oxidized cladding sections were then welded to the 304SS feeding tube and hydrogen permeation tests were subsequently conducted twice for each oxidized sample cladding to exam the repeatability of the results. The maximum and minimum hydrogen leakage from the 304SS feeding tube in Fig. 11 was predicted based on the 'effective hydrogen permeability' discussed in Section 3.1. The hydrogen flux on the surface was first obtained by using Eq. 2, given surface area, temperature, thickness, and hydrogen pressure at any time point of the feeding tube. The total hydrogen content change was the 
integral of the flux over the duration of each constant temperature regime via the trapezoidal method. It is apparent that the measured hydrogen content change in the system for all these four tests are comparable to the predicted values for the feeding tube, implying that the hydrogen is leaking out of the 304SS feeding tube rather than the sample cladding. Note that the detection limit of the current system was limited to the maximum amount of hydrogen released from the 304SS feeding tube. After these tests were conducted, it became clear that the alumina layer was an effective barrier to tritium release, and the system failed to detect any release beyond that from the feeding tube. The adhesion and continuation of the $\mathrm{Al}_{2} \mathrm{O}_{3}$ layer fell out of the scope of this paper and weren't discussed. In this manner, the effectiveness of the alumina layer on the surface of $\mathrm{FeCrAl}$ alloys was crudely demonstrated but was not quantified.

\subsection{In-pile chemical evolution of the FeCrAl cladding surface}

Section 4.2 suggests that incorporating an alumina layer on the surface of the FeCrAl cladding could dramatically reduce tritium transport away from the fuel through this boundary. Note that under normal operating conditions in the high-temperature and highpressure water environments of LWRs, alumina does not form on the surfaces of these alloys. In fact, temperatures of at least $900^{\circ} \mathrm{C}$ are necessary to form protective $\alpha$ - alumina on the surfaces of these alloys in oxidizing environments [41-43]. Under normal operating conditions, the oxide film on the outer surface is instead a sub-micrometer $(\mathrm{Fe}, \mathrm{Cr})_{2} \mathrm{O}_{3}$ type [44], which could also impact the tritium transportation and needs further investigation. One possibility for enabling alumina accumulation on the cladding surfaces (inner or outer) is pre-oxidation at high temperature before fuel pellet insertion. However, 
in the case of the outer surface, dissolution of the alumina layer in contact with the water coolant is a possibility and requires careful examination [44].

However, even if intentional preoxidation of the cladding inner surface is not carried out, alumina can be expected to form once the cladding is in contact with the fuel. To quantify the thermodynamic driving force for such an evolution, one can compare the equilibrium oxygen activity between the $\mathrm{UO}_{2}$ fuel pellet and a model Fe-15Cr-5Al alloy. Figure 12 provides this comparison, showing that the oxygen activity is lowest in equilibrium with the FeCrAl alloy. The equilibrium line for the alloy was calculated using the CALPHAD method [45]. Therefore, it is expected that even in contact with unirradiated $\mathrm{UO}_{2}$, the FeCrAl alloy will oxidize. However, given the fixed amount of oxygen in the system, this reaction will rapidly stop, since the oxygen activity in $\mathrm{UO}_{2}$ drops rapidly as the $\mathrm{O} / \mathrm{M}$ ratio in the compound is reduced. However, as irradiation proceeds, oxygen activity tends to increase in the pellet as a result of the fission of uranium, as experimentally measured by Walker et al. [46], and predicted by Piro et al. [47]. This in turn increases the driving force for oxidation of the internal surface of the $\mathrm{FeCrAl}$ alloy to form alumina.

Contact between fuel pellets and the inner cladding surface is unavoidable during normal LWR operating conditions. But the mitigation of tritium permeation throughout the FeCrAl fuel cladding cannot completely rely on alumina formation in this way, considering the uncertainties related to the heterogeneity of the oxygen activity of the fuel pellets and the onset and the area of fuel-clad contacts. Therefore, it is more 
practical to treat this alumina pathway as a supplement to other, more robust mitigation strategies.

Considering the fact that $\mathrm{Al}_{2} \mathrm{O}_{3}$ is an effective tritium barrier and the primary coolant could dissolve the oxide layer on the outer clad surface, a dense and stable $\mathrm{Al}_{2} \mathrm{O}_{3}$ layer on the inner clad surface could be a potential solution, which is being investigated. Note that this option is only based on the viewpoint of reducing tritium amount and the economy and compatibility to current reactors must be put in considerations for a really practical solution.

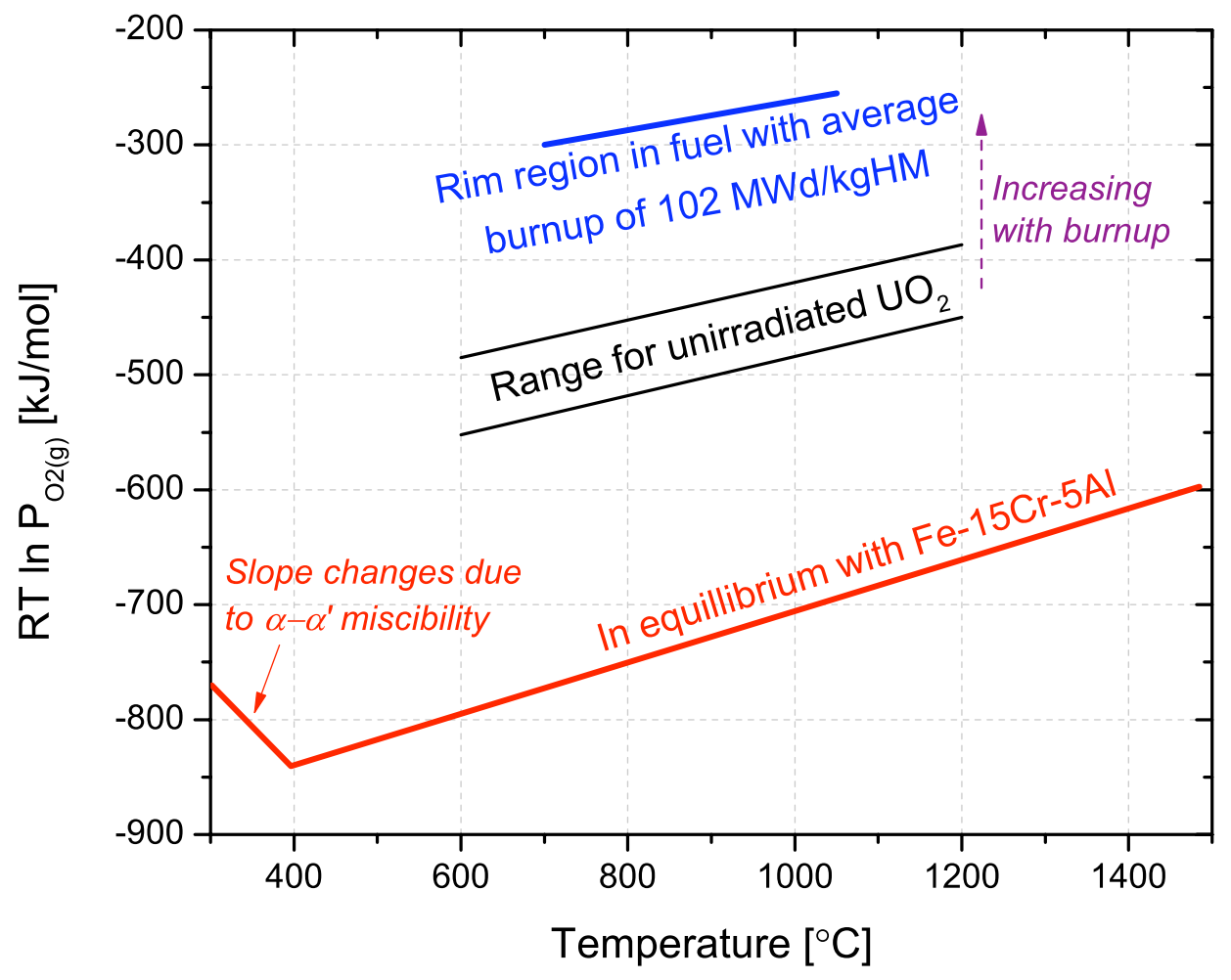


Fig. 12. Oxygen activity in equilibrium with Fe15Cr5Al as calculated by the CALPHAD method. Also shown are oxygen activity in unirradiated $\mathrm{UO}_{2}$ and in the outer (rim) region of a high burnup fuel pellet

[46].

\section{Conclusion}

The hydrogen permeability of model FeCrAl alloys was determined using a static permeation test station. The hydrogen permeation activation energy in FeCrAl alloys was found to increase with increasing chromium content, consistent with previous studies. Given the potential application of FeCrAl alloys in LWR fuel cladding, a 1-dimensional steady state diffusion-controlled model was developed to describe the tritium pathway throughout the cladding and to predict the tritium inventory inside the primary coolant of LWRs using FeCrAl as the fuel cladding material. The results indicated that strategies are needed to mitigate the permeation of tritium generated inside the fuel throughout the cladding. A straightforward externally applied tritium barrier (i.e., alumina) was tested and found to be effective. Furthermore, thermodynamic calculations showed that alumina could form when the fuel pellets are in contact with the inner surface of the fuel cladding. More investigation is necessary to develop an effective, economical tritium barrier on the surface of a FeCrAl fuel cladding before FeCrAl-clad fuels cladding can be practically applied.

\section{Acknowledgements}


The authors would like to extend their gratitude to Dr. Yukinori Yamamoto for providing FeCrAl alloys for this research as well as Theodore Besmann for helping with the thermodynamics calculations. Thoughtful discussions and guidance received from Kevin Field and Bruce Pint at ORNL are also gratefully acknowledged. The work presented in this paper was funded by the U.S. Department of Energy's office of Nuclear Energy, Advanced Fuel Campaign of the Fuel Cycle R\&D program.

\section{References}

1. K.A. Terrani, S.J. Zinkle, and L.L. Snead, J. Nucl. Mater. 448 (2014) 420-435.

2. B.A. Pint, K.A. Unocic, and K.A. Terrani, The effect of steam on the high temperature oxidation behavior of alumina-forming alloys, in Microscopy of Oxidation 9. 2014: Nottingham, UK.

3. B.A. Pint, et al., J. Nucl. Mater. 440 (2013) 420-427.

4. S.J. Zinkle, et al., J. Nucl. Mater. 448 (2014) 374-379.

5. A. Strasser, et al., An Evaluation of Stainless Steel Cladding for Use in Current Design LWRs, NP-2642. 1982, Electric Power Research Institute: Palo Alto, CA.

6. K.E. Moore and W.A. Young, J. Nucl. Mater. 27 (1968) 316-324.

7. Wang, W.E. and D.R. Olander, Journal of American Ceramic Society 78 (1995) $3323-3328$.

8. N. Dupin, I. Ansara, C. Servant, C. Toffolon, C. Lemaignan, J.C. Brachet, J. Nucl. Mater. 275 (1999) 287-295.

9. J.H. Austin, T.S. Elleman, K. Verghese, J. Nucl. Mater. 51 (1974) 321-329. 
10. S. Yamanaka, T. Nishizaki, M. Uno, M. Katsura, Journal of Alloys and Compounds 293-295 (1999) 38-41.

11. Causey, R.A., R.A. Karnesky, and C.S. Marchi, Comprehensive Nuclear Materials 4 (2012) 511-549.

12. E. Hashimoto and T. Kino, J. Nucl. Mater. 133 \& 134 (1985) 289-291.

13. J.K. Gorman and W.R. Nardella, Vacuum 12 (1962) 19-24

14. J.T. Bell, J.D. Redman, and H.F. Bittner, Journal of Materials for Energy Systems 1 (1979) 55-59.

15. T. Tanabe, Y. Yamanishi, K. Sawada and S. Imoto, J. Nucl. Mater. $122 \& 123$ (1984) 1568-1572.

16. D.J. Mitchell and E.M. Edge, Journal of Applied Physics 57 (1985) 5226-5235.

17. T.P. Perng and C.J. Altstetter, Acta Metall. 34 (1986) 1771-1781.

18. D.M. Grant, D.L. Cummings and D.A. Blackburn, J. Nucl. Mater. 149 (1987) $180-191$.

19. X. Sun, J. Xu and Y. Li, Materials Science and Engineering A114 (1989) 179187.

20. T. Shiraishi, M. Nishikawa, T. Yamaguchi, K. Kenmotsu, J. Nucl. Mater. 273 (1999) 60-65.

21. C. San Marchi, B.P. Somerday, S.L. Robinson, International Journal of Hydrogen Energy 32 (2007) 100-116.

22. E.H. Van Deventer and V.A. Maroni, J. Nucl. Mater. 113 (1983) 65-70.

23. J.S. Georgiev and L.A. Anestiev, J. Nucl. Mater. 249 (1997) 133-141.

24. P. Jung, J. Nucl. Mater. 238 (1996) 189-197. 
25. P.J. McGuiness, M. Cekada, V. Nemanic, B. Zajec, and A. Recnik, Surface \& Coatings Technology 205 (2011) 2709-2713.

26. I. Penalva, G. Alberro, J. Aranburu, F. Legarda, J. Sancho, R. Vila, C.J. Ortiz, J. Nucl. Mater. 442 (2013) S719-S722.

27. A.D. LeClaire. Diffus Defect Data 34 (1983) 1-35.

28. K.A. Terrani, M. Balooch, D. Wongsawaeng, S. Jaiyen, D.R. Olander, J. Nucl. Mater. 397 (2010) 61-68.

29. Y.I. Wang, J.Y. Suh, Y.S. Lee, J.H. Shim, E. Fleury, Y.W. Cho, S.U. Koh, Journal of Membrane Science 436 (2013) 195-201.

30. Y. Yamamoto, B.A. Pint, K.A. Terrani, K.G. Field, Y. Yang, L.L. Snead, submitted to J. Nucl. Mater. (2015)

31. K.G. Field, X. Hu, K. Littrell, Y. Yamamoto, R. Howard, and L.L. Snead, ORNL/TM-2014/451.

32. X. Hu, K.A. Terrani, B.D. Wirth, J. Nucl. Mater. 448 (2014) 87-95.

33. J.J. Kearns, J. Nucl. Mater. 43 (1972) 330-338.

34. S. Yamanaka, K. Higuchi, M. Miyake, Journal of Alloys and Compounds 231 (1995) 503-507.

35. L. Dolle, D. Leger, E. Roth, B. Houdaille, Proceedings of the 1980 Topical Meeting on Tritium, ANS.

36. J. Katakura, JENDL FP Decay Data File 2011 and Fission Yields Data File 2011, JAEA-Data/Code 2011-025 (March 2012)

37. R.B. Gregory, L. Crabo, A.J. Percy, A. Rosenberg, Biochemistry 22 (1983) 910917. 
38. J. Crank, P. Nicolson, Math. Proc. Cambridge 43 (1947) 50-64.

39. G.W. Hollenberg, E.P. Simonen, G. Kalinin, A. Terlain, Fusion Engineering and Design 28 (1995) 190-208.

40. J. Konys, W. Krauss, and N. Holstein, Corrosion 67 (2011) 026002.

41. B.A. Pint, K.A. Terrani, M.P. Brady, T. Cheng, K.R. Keiser, J. Nucl. Mater. 440 (2013) 420-427.

42. S.E. Sadique, A.H. Mollah, M.S. Islam, M.M. Ali, M.H.H. Megat, and S. Basri, Oxidation of Metals 54 (2000) 385-400.

43. I.G. Wright, R. Peraldi and B.A. Pint, Materials Science Forum 461-464 (2004) $579-590$.

44. K.A. Terrani, B.A. Pint, Y-J Kim, and L.L. Snead, Proceedings of WRFPM 2014 Sendai, Japan, 2014.

45. H.L. Lukas, S.G. Fries, and B. Sundman, Computational Thermodynamics, the Calphad Method, Cambridge University Press (2007)

46. C.T. Walker et al., J. Nucl. Mater. 345 (2005) 192-205.

47. M.H.A. Piro et al., J. Nucl. Mater., 441 (2013) 240-251. 